\title{
Análise de interações medicamentosas potenciais em pacientes de unidade de terapia intensiva de um hospital de ensino de Sobral
}

\author{
Analysis of potential medicinal interactions in patients in the intensive \\ Therapy unit of a teaching hospital of Sobral
}

Análisis de interacciones medicamentosas potenciales en pacientes de una unidad de terapia intensiva de un hospital de enseñanza de Sobral

Anderson Barros de Sousa ${ }^{1}$, José Davysson Martins Lima Oliveira ${ }^{1}$, André Luiz Cunha Cavalcante², Chrisleny Aguiar Nobre ${ }^{3}$, Olindina Ferreira $\mathrm{Melo}^{3}$, Rafaelly Maria Pinheiro Siqueira ${ }^{3 *}$.

\section{RESUMO}

Objetivo: Analise das interações medicamentosas potenciais em pacientes internados na Unidade de Terapia Intensiva adulta em um hospital de ensino na cidade de Sobral, região norte do Ceará. Metodologia: Tratase de uma pesquisa quantitativa, descritiva e retrospectiva realizada na UTI de um Hospital de ensino localizado no município de Sobral, Ceará, no ano de 2017. Para a coleta de dados foi utilizado um formulário padronizado. Os fármacos utilizados pelos pacientes foram identificados a partir da análise da prescrição médica presente nos prontuários. A análise das interações medicamentosas potenciais ocorreu através das plataformas Micromedex, Drug.com e Medscape. Resultados: Foram analisados 60 prontuários e identificado 639 possíveis Interações Medicamentosas (IMs) com uma frequência de 93,33\%. A IM foi identificada em maior número em indivíduos do sexo masculino com $65 \%$ e $35 \%$ feminino. Essas foram classificadas conforme a gravidade em: moderado 401 (62,75\%), grave $194(30,36 \%)$ e leve $44(6,89 \%)$. Quanto a natureza, as IMs foram classificadas em: farmacodinâmica, 566 (88,58\%), e farmacocinética, $73(11,42 \%)$. A pesquisa identificou a presença de níveis elevados de IMs nas prescrições de pacientes internados na UTI adulta. A IM entre midazolam e fentanil, de gravidade moderada, foi a mais frequente. Conclusão: A pesquisa mostrou que os pacientes da UTI têm alta prevalência de IMs nas prescrições. Na UTI é importante a identificação das IMs buscando reduzir eventos adversos, tempo de internação e custos hospitalares.

Palavras-chave: Farmacovigilância, Medicamentos, Acompanhamento Farmacoterapêutico.

\section{SUMMARY}

Objective: To analyze potential drug interactions in patients admitted to the adult intensive care unit at a teaching hospital in the city of Sobral. Methodology: This is a quantitative, descriptive and retrospective study carried out at the ICU of a teaching hospital located in the municipality of Sobral, Ceará, in the year 2017. A standardized form was used to collect data. The drugs used by the patients were identified from the analysis of the medical prescription present in the medical records. The analysis of potential drug interactions occurred on the Micromedex, Drug.com and Medscape platforms. Results: A total of 60 medical records were analyzed and 639 possible medicinal interactions (MIs) with a frequency of $93.33 \%$ were identified. The MI was identified in greater numbers in male subjects with $65 \%$ and $35 \%$ female. These were classified according to severity in: moderate 401 (62.75\%), severe 194 (30.36\%) and mild 44 (6.89\%). Regarding nature, Mls were classified as: pharmacodynamics, 566 (88.58\%), and pharmacokinetics, $73(11.42 \%)$. The research identified the presence

${ }^{1}$ Farmacêuticos pelo Centro Universitário INTA - UNINTA.

${ }^{2}$ Médico pela Universidade de Fortaleza - UNIFOR.

${ }^{3}$ Farmacêuticas, Docentes do Centro Universitário INTA - UNINTA. *E-mail: rafaellysiqueira@gmail.com 
of high levels of MIs in the prescriptions of patients hospitalized in the adult ICU. MI between midazolam and fentanyl, of moderate severity was the most frequent. Conclusion: The research showed that ICU patients have high prevalence of Mls in prescriptions. In the ICU, it is important to identify Mls seeking to reduce adverse events, hospitalization time and hospital costs.

Key-words: Pharmacovigilance, Drug, Pharmaco-therapeutic follow-up.

\section{RESUMEN}

Objetivo: Análisis de las interacciones medicamentosas potenciales en pacientes internados en la Unidad de Terapia Intensiva adulta en un hospital de enseñanza en la ciudad de Sobral, Ceará, Brasil. Metodología: Se trata de una investigación cuantitativa, descriptiva y retrospectiva realizada en la UTI de un Hospital de enseñanza ubicado en el municipio de Sobral, Ceará, en el año 2017. Se utilizó un formulario estandarizado para la recolección de los datos. Los fármacos utilizados por los pacientes se identificaron a partir del análisis de la prescripción médica en los prontuarios. Se hizo el análisis de las interacciones medicamentosas a través de las plataformas Micromedex, Drug.com y Medscape. Resultados: Se analizaron 60 prontuarios y se identificaron 639 posibles interacciones medicamentosas (IMs) con una frecuencia del 93,33\%. Las IMs se identificaron en mayor número en individuos del sexo masculino con el $65 \%$, y el $35 \%$ femenino, y se clasificaron según la gravedad en: moderado 401 (62,75\%), grave $194(30,36 \%)$ y leve $44(6,89 \%)$. En cuanto a la naturaleza, las IMs se clasificaron en: farmacodinámica, 566 (88,58\%) y farmacocinética, 73 (11,42\%). La investigación identificó la presencia de niveles elevados de IMs en las prescripciones de pacientes internados en la UTI adulta. La IM entre midazolam y fentanilo, de gravedad moderada, fue la más frecuente. Conclusión: La investigación mostró que los pacientes de la UTI tienen alta prevalencia de IMs en las prescripciones. Es importante identificar a las IMs en la UTI buscando reducir eventos adversos, tiempo de internación y costos hospitalarios.

Palabras clave: Farmacovigilancia, Medicamentos, Acompañamiento Farmacoterapéutico.

\section{INTRODUÇÃO}

As Unidades de Terapia Intensiva (UTIs) são os setores hospitalares que concentram os pacientes mais críticos ou de alto risco de morte que necessitam de uma atenção especializada (HAMMES JA, et al., 2008). Os pacientes internados em UTI geralmente recebem esquemas com múltiplos fármacos devido às condições clínicas graves. Com isso, apresentam alto risco de ocorrência de interações medicamentosas (IM) devido à complexidade da polifarmacoterapia (VIEIRA LB, et al., 2012; FUERTES DG, et al., 2016).

Interação Medicamentosa (IM) são respostas farmacológicas de um ou mais medicamentos que altera o efeito de outro se administrado concomitantemente ou não (SECOLI SR, 2001; MATOS VTG, et al., 2009). De acordo com Fuchs FC e Wannmacher L (2017); as IMs podem ser classificadas em: farmacêutica, farmacocinética e farmacodinâmica.

Essas IMs podem ser classificadas de acordo com sua origem e o potencial de gravidade. A IM apresenta uma classificação quanto sua gravidade: leve, moderado e grave (AIZENSTEIN ML e TOMASSI MH, 2011). Os profissionais de saúde precisam ficar atentos, pois algumas delas podem levar o paciente a situações de morbimortalidade (JACOMINI LCL e SILVA NA, 2011; CEDRAZ KN e JUNIOR MCS, 2014; HASANLOEI V, et al., 2014).

O farmacêutico de UTI deve possuir habilidades para atuar em diversos seguimentos que envolve 0 medicamento, da prescrição até a administração do medicamento. Assim o farmacêutico pode contribuir com os médicos e fornecendo informações a equipe de enfermagem sobre formas seguras de administrações dos medicamentos que são prescritos, com tudo isso fazendo o acompanhamento da eficiência dos medicamentos (PILAU R, et al., 2013; FIDELES GMA, et al., 2015). 
Portanto, visando contribuir para o conhecimento sobre possíveis interações medicamentosas e reduzir os riscos provenientes do tratamento farmacológico, o presente estudo teve como objetivo identificar as potenciais interações medicamentosas em Unidades de Terapia Intensiva (UTI) de um Hospital da Região Norte do Ceará.

\section{MÉTODOS}

A pesquisa trata-se de um estudo de campo quantitativo, descritivo e retrospectivo que abordou a análise de prontuários dos pacientes internados em UTI de um Hospital de ensino da região norte do Ceará ano de 2017. A amostra do estudo corresponde a 60 prontuários do período de janeiro a dezembro de 2017 referentes à pacientes internados na UTI adulta do bloco da emergência.

Inicialmente, todos os prontuários de pacientes foram inseridos na amostra do estudo. Foram excluídos os prontuários de pacientes com idade inferior a 18 e superior a 60 anos, devendo apresentar prescrição de no mínimo 5 medicamentos.

Para a identificação das interações medicamentosas foram usadas as plataformas Micromedex, Drug.com, Medscape. As informações obtidas foram tabuladas no software Excel® 2016.

A pesquisa foi realizada de acordo com a Resolução do Conselho Nacional de Saúde - o 466 de 12 de dezembro de 2012 e suas diretrizes e normas que regulamentam a pesquisa envolvendo seres humanos no Brasil. O projeto de pesquisa foi submetido ao Comitê de Ética através da plataforma Brasil e obteve parecer de aprovação no 2.699.824.

\section{RESULTADOS}

Analisaram-se os prontuários dos pacientes internados na UTI adulta durante o ano de 2017. Respeitando os critérios de inclusão, 60 prontuários foram selecionados.

Os prontuários foram categorizados quanto ao sexo dos pacientes. No total de 60 prontuários, 39 (65\%) eram do sexo masculino e 21 (35\%) do sexo feminino, como mostra a Tabela 1.

Tabela 1 - Categorização quanto ao sexo de 60 prontuários de pacientes internados na UTI adulta de um hospital de ensino na cidade de Sobral-Ceará, no ano de 2017.

\begin{tabular}{ccc}
\hline SEXO & FREQUÊNCIA ABSOLUTA & FREQUÊNCIA RELATIVA (\%) \\
\hline Feminino & 21 & 35 \\
Masculino & 39 & 65 \\
Total & 60 & 100 \\
\hline
\end{tabular}

Fonte: dados da pesquisa.

Foram identificadas 639 interações medicamentosas potenciais, sendo a mais notificada à interação entre o midazolam e o fentanil, detectada em 29 prontuários; seguindo das interações entre bromoprida e fentanil, bromoprida e midazolam, ambas detectadas em 25 prontuários; midazolam e omeprazol ocorreram em 23 prontuários; midazolam e noradrenalina foram observadas em 21 prontuários. A Tabela 2 mostra as 20 IMs mais identificadas, junto com as classes e gravidade.

O Gráfico 1 apresenta a gravidade das IMs identificadas. Dentre as 639 IMs, 401 (62,75\%) foram classificadas como moderadas, 194 (30,36\%) são do tipo grave e $44(6,89 \%)$ são do tipo leve. 
Tabela 2 - Princípios ativos, classes terapêuticas, gravidade e número de prontuários com a identificação da respectiva Interação Medicamentosa em pacientes internados em UTI adulta de um hospital de ensino na cidade de Sobral-Ceará, no ano de 2017.

\begin{tabular}{|c|c|c|c|}
\hline Fármacos & Classes terapêuticas & Gravidade & № \\
\hline midazolam $\leftrightarrow$ fentanil & benzodiazepínico↔opiode & moderado & 29 \\
\hline bromoprida $\leftrightarrow$ fentanil & antiemético $\leftrightarrow$ opiode & grave & 25 \\
\hline bromoprida $\leftrightarrow$ midazolam & antiemético $\leftrightarrow$ benzodiazepínico & grave & 25 \\
\hline midazolam $\leftrightarrow$ omeprazol & $\begin{array}{c}\text { benzodiazepínico } \leftrightarrow \text { inibidor da bomba de } \\
\text { prótons }\end{array}$ & moderado & 23 \\
\hline midazolam $\leftrightarrow$ noradrenalina & benzodiazepínico $\leftrightarrow$ vasopressor & grave & 21 \\
\hline furosemida $\leftrightarrow$ dipirona & diurético de alça $\leftrightarrow$ analgésico/antipirético & grave & 16 \\
\hline omeprazol↔fenitoína & inibidor da bomba de prótons $\leftrightarrow$ antiepiléptico & moderado & 15 \\
\hline $\begin{array}{c}\text { noradrenalina } \leftrightarrow \text { insulina } \\
\text { regular }\end{array}$ & vasopressor $\leftrightarrow$ antidiabético & moderado & 15 \\
\hline furosemida $\leftrightarrow$ midazolam & diurético de alça $\leftrightarrow$ benzodiazepínico & moderado & 15 \\
\hline furosemida $\leftrightarrow$ noradrenalina & diurético de alça $\leftrightarrow$ vasopressor & moderado & 15 \\
\hline $\begin{array}{c}\text { cloreto de } \\
\text { potássio } \leftrightarrow \text { noradrenalina }\end{array}$ & eletrólito $\leftrightarrow$ vasopressor & grave & 14 \\
\hline bromoprida $\leftrightarrow$ fenitoina & antiemético↔antiepiléptico & moderado & 13 \\
\hline fentanil $\leftrightarrow$ fenitoina & opiode $\leftrightarrow$ antiepiléptico & grave & 12 \\
\hline furosemida $\leftrightarrow$ insulina $r$ & diurético de alça $\leftrightarrow$ antidiabético & moderado & 12 \\
\hline furosemida $\leftrightarrow$ omeprazol & $\begin{array}{c}\text { diurético de alça↔inibidor da bomba de } \\
\text { prótons }\end{array}$ & moderado & 12 \\
\hline furosemida $\leftrightarrow$ hidrocortisona & diurético de alça↔corticóide & moderado & 11 \\
\hline furosemida $\leftrightarrow$ fentanil & diurético de alça $\leftrightarrow$ opiode & moderado & 10 \\
\hline $\begin{array}{c}\text { ácido } \\
\text { acetilsalicílico } \leftrightarrow \text { furosemida }\end{array}$ & salicilato $\leftrightarrow$ diurético de alça & grave & 10 \\
\hline ácido acetilsalicílico↔dipirona & salicilato↔analgésico/antipirético & grave & 10 \\
\hline furosemida $\leftrightarrow$ cefepime & diurético de alça $\leftrightarrow$ antimicrobiano & moderado & 10 \\
\hline
\end{tabular}

Fonte: dados da pesquisa.

O estudo também identificou a natureza envolvida nas IMs e classificou em: farmacocinética e/ou farmacodinâmica conforme classificação apresentada por Fuchs FC e Wannmacher L (2017).

O Gráfico 2 apresenta a natureza envolvida na IM com 566 (88,58\%) IMs farmacodinâmicas e $73(11,42 \%)$ com natureza farmacocinética.

O Gráfico 3 mostra uma subclassificação das IMs farmacodinâmicas e farmacocinéticas. As IMs farmacodinâmicas são subclassificadas em: ação e efeito, contabilizando $172(26,92 \%)$ e $394(61,66 \%)$ IMs respectivamente. A subclassificação das IMs farmacocinéticas identificadas foram: absorção, metabolização e eliminação, totalizando 4 (0,62\%), $62(9,70 \%)$ e $7(1,10 \%)$ IMs respectivamente nessa ordem. 
Gráfico 1 - Classificação das interações medicamentosas quanto a gravidade em 60 prontuários de pacientes internado em UTI adulta de um hospital de ensino na cidade de Sobral-Ceará, no ano de 2017.

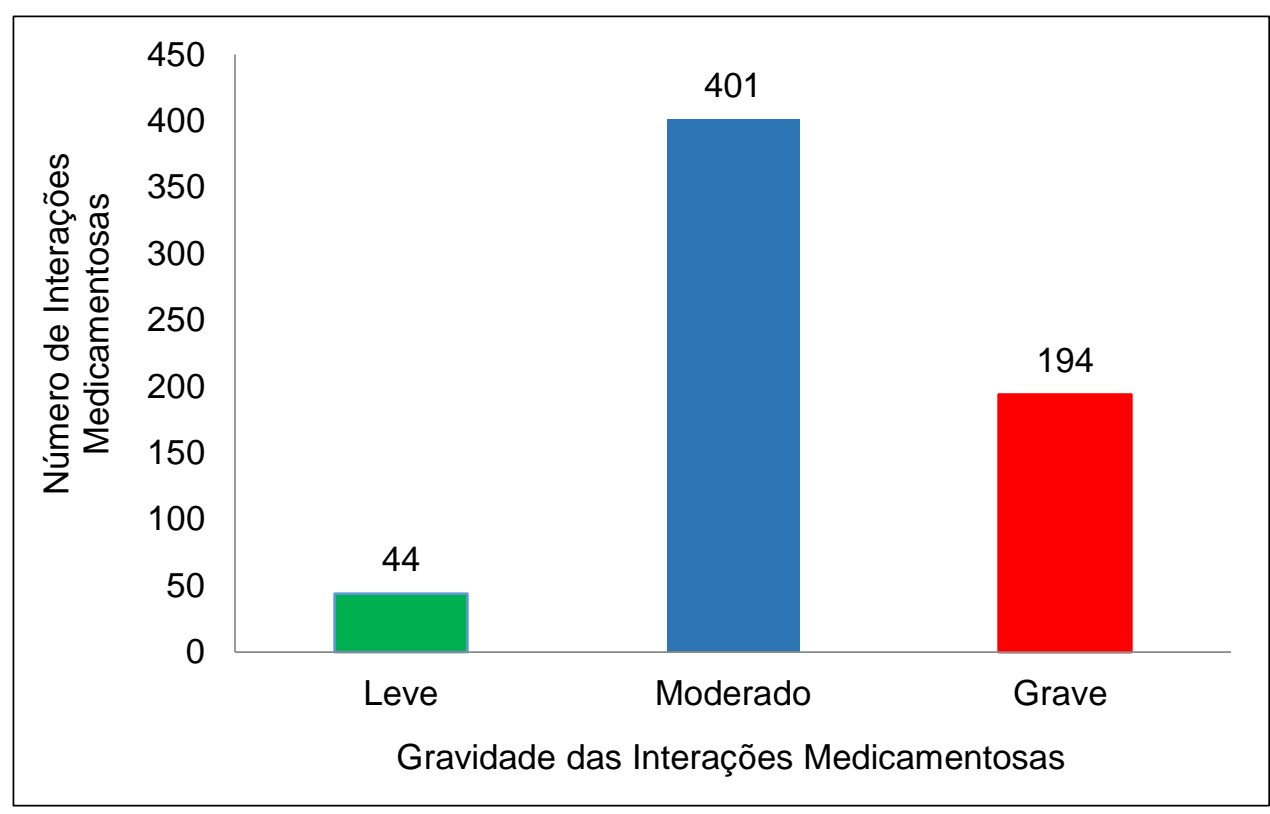

Fonte: dados da pesquisa.

Gráfico 2 - Classificação das interações medicamentosas quanto a natureza em 60 prontuários de pacientes internado em UTI adulta de um hospital de ensino na cidade de Sobral-Ceará, no ano de 2017.

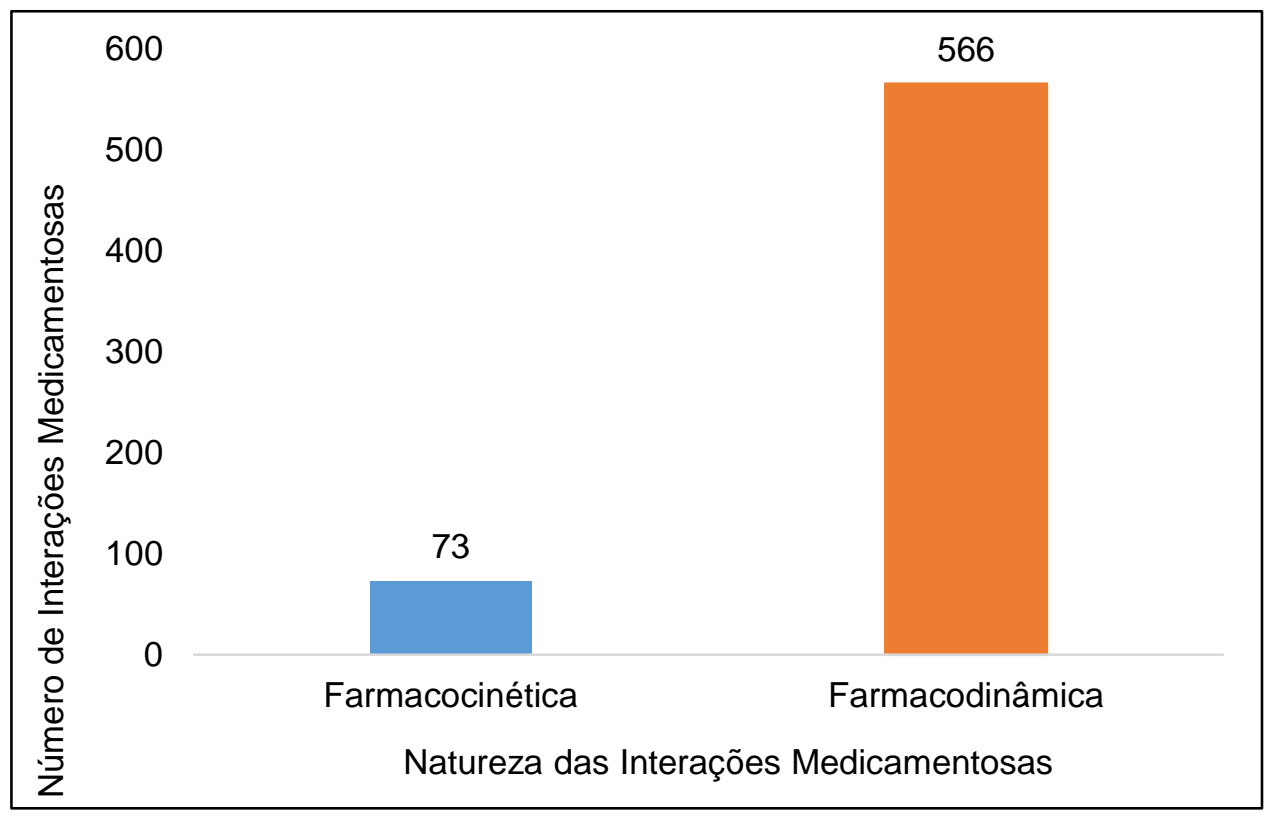

Fonte: dados da pesquisa. 
Gráfico 3 - Subclassificação das interações medicamentosas Farmacocinéticas e Farmacodinâmicas em 60 prontuários de pacientes internado em UTI adulta de um hospital de ensino na cidade de Sobral-Ceará, no ano de 2017.

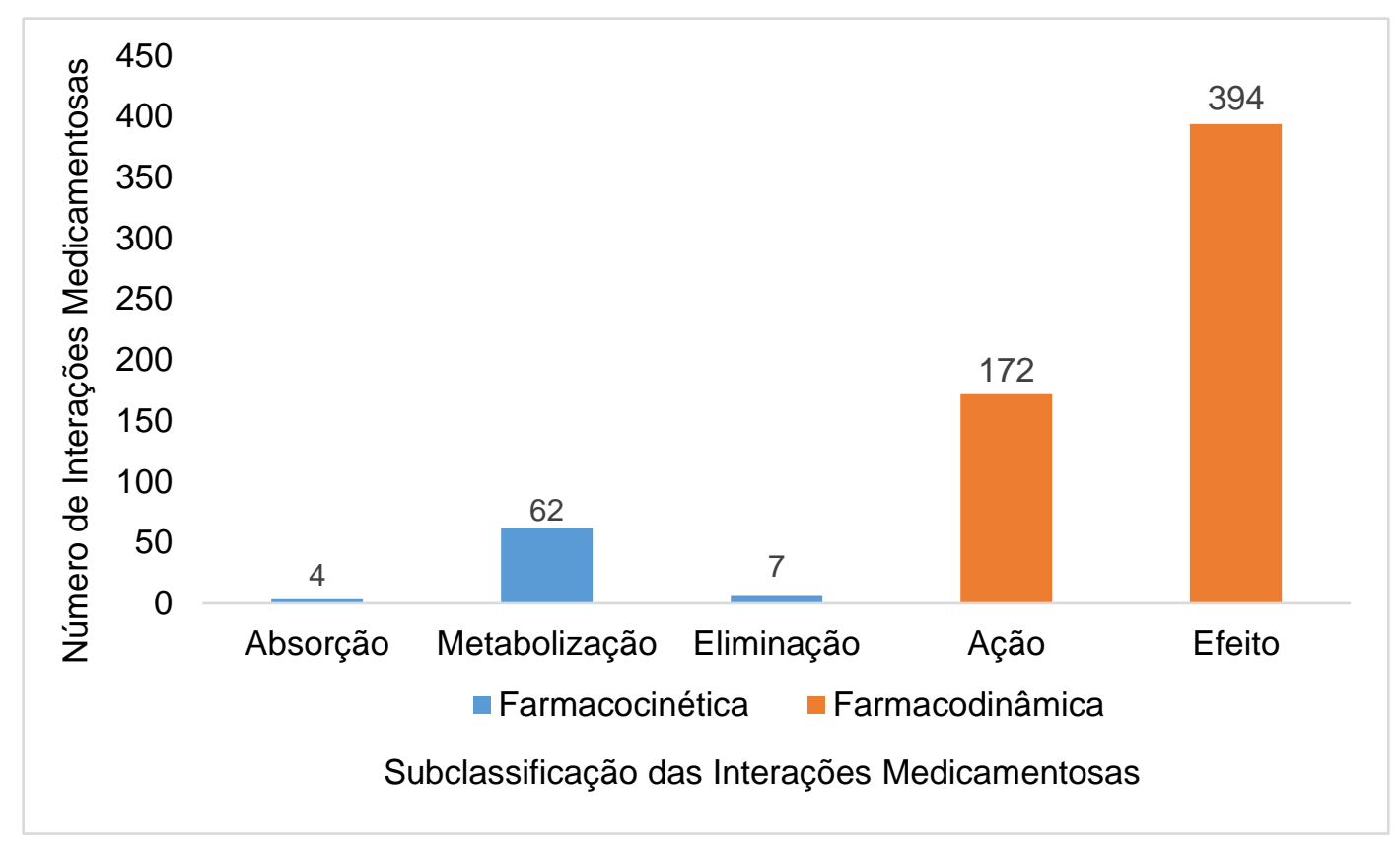

Fonte: dados da pesquisa.

\section{DISCUSSÃO}

A característica obtida frente ao sexo apresentou um predomínio de interações medicamentosas potenciais em pacientes do sexo masculino. Segundo a política nacional de atenção integral à saúde do homem do Ministério da Saúde, o homem julga-se invulnerável, o que acaba por contribuir para a negligência ao autocuidado e maior exposição a situações de risco. O homem é mais vulnerável à violência, seja como autor, seja como vítima e as agressões sofridas são mais graves e demandam maior tempo de internação, em relação às sofridas pelas mulheres. Esses fatos levam a inferir que quanto mais frequente e maior o tempo de internação, maior a probabilidade de estar vulnerável às IMs (SCHWARZ E, et al., 2012).

No presente estudo, observamos predominância do sexo masculino (65\%) conforme demonstrado no gráfico 1. Em estudos realizados sobre IMs em outros estabelecimentos de saúde, a frequência era de 56,4\% a $66,65 \%$ do sexo masculino e $33,35 \%$ a $43,6 \%$ do sexo feminino (LIMA REF e CASSIANI SHB, 2009; CEDRAZ KN e JUNIOR MCS, 2014; SCRIGNOLI CP, et al., 2016).

Para Reis AMM (2009); são fatores sujeitos a um alto índice de interações medicamentosas em UTI: paciente em estado geral grave, a gravidade da doença, tempo de internação e idade do paciente. A quantidade de medicamentos prescritos é um forte indicador de risco, pois quanto maior o número de medicamentos maior a possibilidade de interações entre esses (VIEIRA et al., 2012; FUERTES et al., 2016).

Os resultados obtidos corroboram com os demais achados de modo a ressaltar a importância das IM em UTIs. A identificação de IMs variou de $76 \%$ a $80 \%$ em população atendida em UTIs de diferentes regiões do Brasil, onde as prescrições apresentavam pelo menos uma interação (MELGAÇO TB, et al., 2011; CARIBÉ RA, et al., 2013; OLIVEIRA-PAULA GH, et al., 2014). A frequência de IM identificada nesta pesquisa foi superior aos resultados obtidos por demais autores, alcançando $93,33 \%$ dos prontuários analisados. Essa variação pode ser explicada através do uso de diferentes softwares empregados no reconhecimento das IMs, de acordo com a especificidade e sensibilidade da plataforma analisada. 
Com a quantidade de IMs encontradas, 639, em apenas 60 prontuários da UTI adulta, ressaltam-se a importância desses pacientes serem acompanhados diariamente por um profissional habilitado na realização de um acompanhamento farmacoterapêutico junto à equipe multiprofissional contribuindo para um melhor prognóstico.

A natureza envolvida na presente pesquisa que prevaleceu foi à farmacodinâmica com $88,58 \%(566)$ e farmacocinética com $11,42 \%$ (73), isso mostra uma variação de IM com os demais estudos. Em outro estudo realizado por Moura CS (2010); a predominância do tipo da natureza da IM foi $67 \%$ do tipo farmacocinética. O estudo da Nobrega RC (2013); obteve como interação mais prevalente a do tipo farmacocinética com $67,6 \%, 21,6 \%$ de farmacodinâmica e interação desconhecida foram de 10,8\%. Já o estudo de Carvalho MF et al. (2013); observou-se o predomínio de IM do tipo farmacodinâmica com 42,2\%, 39,6\% de farmacocinética e 18,2\% foram IM que envolveu ação mista.

Dentre os resultados, a associação entre o midazolam e fentanil ocorreu com maior frequência, estando presente em 29 dos 60 prontuários analisados. Essa interação farmacodinâmica moderada tem como fundamento o sinergismo farmacológico na sedação através da utilização de um opiode (fentanil) associado com um benzodiazepínico (midazolam). Resultados semelhantes dessa interação podem ser observado nos estudos realizado por Lima REF e Cassiani SHB (2009), Oliveira-Paula GH et al. (2014); Rocha PCF et al. (2014). A associação desses dois fármacos tem efeito aditivo sobre o Sistema Nervoso Central (SNC), podendo resultar em depressão respiratória. São muito utilizados para conforto e alívio da dor, porém podem agravar o quadro clínico do paciente e necessitar de intervenção médica (LIMA REF e CASSIANI SHB, 2009; MOREIRA MB, et al., 2017).

As interações classificadas como do tipo farmacocinética mais prevalente do estudo ocorreu entre os fármacos midazolam e omeprazol. Essa IM apresenta gravidade moderada e seu mecanismo envolve a elevação nos níveis plasmáticos do fármaco midazolam. Esse aumento está relacionado ao fato do omeprazol atuar como inibidor enzimático, reduzindo os níveis de enzimas envolvidas na metabolização do midazolam e de diversos fármacos (CARVALHO MF, et al., 2013).

Os benzodiazepínicos (BZD) têm o efeito depressor no SNC, uma interação farmacocinética auxilia para a potencialização dos seus efeitos depressores dos BZD. Por favorecer a inibição do seu metabolismo hepático, os inibidores da bomba de prótons, podem promover o aumento dos níveis plasmáticos e causar ataxia e fraqueza muscular por deprimir diferentes regiões do SNC (VIEL AM, et al., 2014).

A IM entre omeprazol e furosemida é do tipo moderado com natureza farmacodinâmica de efeito, onde os inibidores da bomba de prótons podem favorecer ao risco de hipomagnesemia, quando utilizados em concomitância por um período prolongado (SCRIGNOLI CP, et al., 2016).

De acordo com esta pesquisa, quanto à classificação da gravidade das IMs, foi observado um nível elevado de interações do tipo grave, visto que, essa interação apresenta risco à vida do paciente, requerendo intervenção médica.

A gravidade moderada foi a mais prevalente no estudo, ocorre quando há exacerbação da condição de saúde do paciente, sendo necessária a mudança da terapia (NOBREGA RC, 2013).

A pesquisa identificou diferença quantitativa entre a gravidade das IMs. Dentre as IMs identificadas, $62,75 \%$ foram classificadas como gravidade moderada e 30,63\% grave, como mostrado no gráfico 2 . O estudo mostrou semelhança com outros estudos em que foram avaliadas IM em UTIs de diferentes hospitais espalhados pelo Brasil, apresentando frequência de $50 \%$ a $67 \%$ de IMs moderadas e $7 \%$ a $30 \%$ de IMs graves (HAMMES JA, et al., 2008; YUNES LP, et al., 2011; FIRMO BDA, 2014; ROCHA PCF, et al., 2014).

Segundo Almeida SM et al. (2007), Hammes JA et al. (2008) as IMs moderadas ou graves, podem ser controladas por outros meios que não a suspensão, mas sim ajustando a doses e monitorando os possíveis efeitos adversos.

Em algumas circunstâncias, o resultado da IM pode ser necessário, permitindo ao alcance de um efeito potencializado através da redução da dose de um ou ambos os fármacos administrados ao paciente e, desse 
modo, contornando a exacerbação de efeitos adversos. Devendo essa IM ser identificada, estudada, avaliada e monitorada quando aos possíveis riscos-benefícios ao paciente.

O Acompanhamento Farmacoterapêutico (AF) está dentre as atividades inerentes ao farmacêutico para limitar erros com medicação, que vai resultar na eficácia do tratamento e na melhora da qualidade de vida (STURARO D, 2009). Na prática, o farmacêutico assume o compromisso do resultado da terapia medicamentosa de um paciente, assegurando que a terapia minimize ou evite algum problema relacionado com medicamento e traga resultados positivos para o paciente (NUNES LMN, et al., 2012).

\section{CONCLUSÃO}

Constatou-se uma alta frequência de IM potenciais nas prescrições de pacientes internados em UTI no período compreendido entre janeiro a dezembro de 2017. Embora o número de episódios de IM moderadas tenha prevalecido, observou-se um grande número de episódios de IM graves. Torna-se importante a utilização de sistemas informatizados e a promoção da atenção multiprofissional ao paciente, a fim de reduzir a ocorrência desse evento. A presença do farmacêutico na UTI pode permitir análises das prescrições hospitalares com objetivo de identificar as possíveis interações e intervir junto ao médico e enfermeiro formando um elo de cuidado ao paciente em estado crítico, agregando segurança para a terapia do paciente.

\section{AGRADECIMENTOS}

Ao Hospital e Maternidade referência como campo de ensino de diferentes categorias profissionais da cidade de Sobral, Ceará, por ceder o espaço e a possibilidade da realização da pesquisa, a toda equipe do Serviço de Arquivo Médico e Estatística (SAME), pela receptividade e colaboração.

\section{REFERÊNCIAS}

1. ALMEIDA SM, et al. Prevalência e classificação de interações entre medicamentos dispensados para pacientes em terapia intensiva. Revista Einstein, v.5, n.4, v.347-351, 2007.

2. AIZENSTEIN ML, TOMASSI MH. Problemas relacionados a medicamentos; reações adversas a medicamentos e erros de medicação: a necessidade de uma padronização nas definições e classificações. Revista de Ciências Farmacêuticas Básica e Aplicada, 2011; 32(2): 169-173.

3. CARIBÉ RA, et al. Potenciales interacciones medicamentosas en pacientes com sepsis internado em la unidade de terapia intensiva. Farmácia Hospitalaria, 2013; 37(5): 383-387.

4. CARVALHO MF, et al. Fatores de risco para interações medicamentosas: uma revisão da produção científica. Revista Arquivos de Ciências da Saúde, 2013; 20(4): 123-127.

5. CEDRAZ KN, JUNIOR MCS. Identificação e caracterização de interações medicamentosas em prescrições médicas da unidade de terapia intensiva de um hospital público da cidade de Feira de Santana, BA. Revista da Sociedade Brasileira de Clínica Médica, 2014; 12(2):124-130.

6. FIDELES GMA, et al. Recomendações farmacêuticas em unidade de terapia intensiva: três anos de atividades clínicas. Revista Brasileira de Terapia Intensiva, 2015; 27(2): 149-154.

7. FIRMO BDA. Interações medicamentosas potencias em pacientes da unidade de terapia intensiva de um Hospital Filantrópico. Trabalho de Conclusão de Curso (Graduação em Farmácia) - Universidade Estadual da Paraíba, Campina Grande, 2014; 35p.

8. FUCHS FC, WANNMACHER L. Farmacologia Clínica e Terapêutica, 5.ed. Rio de Janeiro: Guanabara Koogan, 2017; 852p.

9. FUERTES DG, et al. QT longo e torsades de pointes induzidos por fármacos em pacientes idosos polimedicados. Arquivo Brasileiros de Cardiologia, 2016; 106(2): 156-159.

10. HAMMES JA, et al. Prevalência de potenciais interações medicamentosas droga-droga em unidades de terapia intensiva. Revista Brasileira de Terapia Intensiva, 2008; 20(4): 349-354. 
11. HASANLOEI V, et al. Drug-Drug interactions prevalence in intensive care unit patients of a university hospital in Iran. Bulletin of Environment, Pharmacology and Life Sciences, 2014; 3(7): 87-91.

12. JACOMINI LCL, SILVA NA. Interações medicamentosas: uma contribuição para uso racional de imunossupressores sintéticos e biológicos. Revista Brasileira de Reumatologia, 2011; 51(2): 161-174.

13. LIMA REF, CASSIANI SHB. Interações medicamentosas potencias em pacientes de unidade de terapia intensiva de um hospital universitário. Revista Latino-Americana de Enfermagem, 2009; 17(2): 81-87.

14. MATOS VTG, et al. Avaliação das interações medicamentosas em prescrições hospitalares de pacientes sob uso de anti-hipertensivos. Latin American Journal of Pharmacy, 2009; 28(4): 501-506.

15. MELGAÇO TB, et al. Polifamácia e ocorrênca de possíveis interações medicamentosas. Revista Einstein, 2011; 6(4): $110-118$.

16. MOREIRA MB, et al. Potencias interações de medicamentos intravenosos em terapia intensiva. Revista da Escola de Enfermagem da USP, 2017; 51: e03233.

17. MOURA CS. Interações medicamentosas em pacientes hospitalizados: exposição, relação com indicadores de internação e intervenção. Tese (Doutorado em Ciências Farmacêuticas), Universidade Federal de Minas Gerais, Belo Horizonte, 2010; 133p.

18. NOBREGA RC. Avaliação de interações medicamentosas potenciais envolvendo antimicrobianos em unidade de terapia intensiva de um hospital público de ensino de João Pessoa - PB. Trabalho de Conclusão de Curso (Graduação em Farmácia), Universidade Federal da Paraíba, João Pessoa, 2013; 42p.

19. NUNES LMN, et al. Acompanhamento farmacoterapêutico de pacientes diabéticos tipo 2 e fatores de risco associados. Revista Brasileira de Farmácia, 2012; 93(2): 196-203.

20. OLIVEIRA-PAULA GH, et al. Interações medicamentosas potencias em unidade de terapia intensiva de um hospital do sul do Brasil. Semina: Ciências Biológicas e da Saúde, 2014; 35(2): 21-30.

21. PILAU R, et al. Atuação do farmacêutico clinico em unidade de terapia intensiva adulto: Uma revisão da literatura. Revista Brasileira de Farmácia Hospitalar e Serviços de Saúde, 2013; 5(1): 19-24.

22. REIS AMM. Fatores Associados às Interações Medicamentosas em uma Unidade de Terapia Intensiva. Tese (Doutorado em Ciências) - Programa Enfermagem Fundamental, Universidade de São Paulo, Ribeirão Preto, 2009; 195 p.

23. ROCHA PCF, et al. Prevalência de potenciais interações medicamentosas em uma unidade de terapia intensiva de Manaus- AM. Revista Brasileira de Farmácia, 2014; 95(4): 909-923.

24. SCHWARZ E, et al. Política de saúde do homem. Revista Saúde Pública, 2012; 2(3): 9-18.

25. SCRIGNOLI CP, et al. Interações medicamentosas entre fármacos mais prescritos em unidade de terapia intensiva adulta. Revista Brasileira de Farmácia Hospitalar e Serviços de Saúde, 2016; 7(2): 26-30.

26. STURARO D. A importância do acompanhamento farmacoterapêutico em pacientes onco-hematológicos. Revista Brasileira de Hematologia e Hemoterapia, 2009; 31(3): 124.

27. SECOLI SR. Interações medicamentosas: fundamentos para a prática clínica da enfermagem. Revista da Escola de Enfermagem, 2001; 35(1): 28-34.

28. VIEIRA LB, et al. Interações medicamentosas potenciais em pacientes de unidades de terapia intensiva. Revista de Ciências Farmacêuticas Básica e Aplicada, 2012; 33(3): 401-408.

29. VIEL AM, et al. Interações medicamentosas potenciais com benzodiazepínicos em prescrições médicas de pacientes hospitalizados. Revista de Ciência Farmacêutica Básica e Aplicada, 2014; 35(4): 589-596.

30. YUNES LP, et al. Principais interações medicamentosas em pacientes da uti-adulto de um hospital privado de minas gerais. Revista Brasileira de Farmácia Hospitalar e Serviços de Saúde, 2011; 2(3): 23-26 